\title{
BAYLE HISTORIEN ET CRITIQUE DU MATÉRIALISME DANS LE DICTIONNAIRE
}

\author{
Jean-Luc Solère* \\ solere@bc.edu
}

RESUMO Bayle afirma que os atomistas deveriam se manter fiéis a uma curiosa tese atribuída ao fundador do atomismo, Demócrito, a saber, que os átomos possuem uma alma. De fato, mostra Bayle por diversos argumentos, é impossível que o pensamento emerja da matéria. Assim o atomismo deve considerar o pensamento como presente em cada um dos átomos. Nesta mesma linha, Bayle mostra que, mais geralmente, todo materialismo consequente deve ser um panpsiquismo: todas as partes da matéria devem pensar, e pensar sempre. Mas isto conduz a consequeências dificilmente aceitáveis, como cadáveres pensantes. Além disto, o conselho que ele dá aos materialistas (seguir Demócrito) é uma medida que ele crê ser de fato inaplicável, já que, segundo Bayle, o pensamento é incompativel com a extensão, sendo suas propriedades opostas e cada substância só possuindo um atributo. O que Bayle busca então fazer, nesta fantasia histórica sobre Demócrito, é reduzir o materialismo a uma posição absurda.

Palvras-chave alma, atomismo, atributo, cartesianismo, corpo, Demócrito, Epicuro, Dicearco, extensão, Locke (John), materialismo, modos, pampsiquismo, pensamento, substância, Toldand (John)

RÉSUMÉ Bayle affirme que les atomistes auraient dî rester fidèles à une curieuse thèse attribuée au fondateur de l'atomisme, Démocrite, à savoir que les atomes ont une âme. En effet, montre Bayle par plusieurs arguments, il est impossible que la pensée émerge de la matière. Dès lors, l'atomisme

* Boston College, Massachusetts. Recebido em 19/08/2009 e aceito em 02/09/2009.

KRITERION, Belo Horizonte, nº 120, Dez./2009, p. 423-436. 
doit se donner d'emblée la pensée comme présente dans chacun des atomes. Dans la même ligne, Bayle montre que, plus généralement, tout matérialisme conséquent doit être un panpsiquismo : toutes les parties de la matière doivent penser, et penser toujours. Mais cela conduit à des conséquences difficilement acceptables, telles que des cadavres pensants. De plus, le conseil qu'il donne aux matérialistes (suivre Démocrite) est une mesure qu'il croit en réalité inapplicable, puisque selon Bayle la pensée est incompatible avec l'étendue, leurs propriétés étant opposées et chaque substance n'ayant qu'un attribut. Ce que Bayle entend donc faire, dans cette fantaisie historique sur Démocrite, est de réduire le matérialisme à une position absurde.

Mots clés ame, atomisme, attribut, cartésianisme, corps, Démocrite, Epicure, Dicéarque, étendue, Locke (John), matérialisme, modes, panpsychisme, pensée, substance, Toland (John)

Incomparable est l'art qu'a Bayle de glisser de l'érudition historique à la discussion philosophique d'actualité. Ses considérations sur les matérialistes, dans le Dictionnaire, en sont l'illustration. Les notices qu'il consacre à quelques auteurs antiques sont pour lui l'occasion de développer une argumentation serrée contre le matérialisme moderne. Voyons comment.

\section{Démocrite et l'âme des atomes}

Dans l'article du Dictionnaire consacré à Épicure, Bayle rappelle que ce n'est pas ce dernier, mais Démocrite, qui est l'inventeur du « système des atomes ». Épicure y introduisit des changements et, au jugement de Bayle, pas toujours à bon escient. En particulier, « ce fut gâter le systême que de ne pas retenir la doctrine de Démocrite touchant l'ame des atômes ». ${ }^{1}$ Cette omission conduit en effet à une impasse. Les âmes n'étant pour Épicure autre chose que la rencontre fortuite d'atomes inanimés, ce sont donc selon lui des corps inanimés qui pensent, informés par le moyen des fameux « eidola», c'est-àdire des images matérielles reçues d'autres corps qui sont aussi la rencontre fortuite de semblables atomes. Or, juge Bayle, «prétendre qu'un assemblage 
d'atômes inanimez peut être une ame, et peut envoier des images qui nous donnent des pensées, c'est se paier d'une hypothese plus confuse que le cahos d'Hesiode $» .^{2}$

Cette thèse est en effet exposée à une critique décisive, que Bayle dit emprunter à Galien par l'intermédiaire de Gassendi :

[Argument 1] Si un atome n'est pas capable de sensibilité, deux ne le sont pas non plus, ni trois, ni quatre, ni une pluralité quelconque, et donc pas davantage une âme constituée d'atomes.

En d'autres termes, ce sorite vise à prouver que la sensibilité ne pourra jamais émerger d'une combinaison d'atomes insensibles, en quelque nombre qu'ils soient. Ainsi que Bayle y fait allusion ensuite, la même objection est faite par Plutarque dans son traité contre l'épicurien Colotès. Expliquons donc le sorite en examinant quelques passages de ce dernier ouvrage.

Selon Épicure, les atomes sont des substances dépourvues de qualités : ils n'ont que des caractéristiques dimensionnelles. De plus, ils sont impassibles du fait de leur dureté. Il s'ensuit, dit Plutarque, qu'aucun composé et aucune qualité ne pourront être engendrés :

Prenons immédiatement l'exemple de ce que vous appelez le chaud : d'où vient-il ? Comment s'est-il ajouté aux atomes, lesquels n'avaient pas de chaleur en arrivant, et que leur réunion n'a pas rendu chauds? En effet, dans le premier cas <i.e. s'ils arrivaient avec de la chaleur>, les atomes seraient dotés d'une qualité; dans l'autre <i.e. si leur réunion les rendait chauds>, ils seraient susceptibles d'être affectés. Or aucune de ces deux conséquences - c'est vous qui l'affirmez - ne convient aux atomes, à cause de leur incorruptibilité. ${ }^{3}$

Aucun changement ne se fera jour, aucune nouvelle propriété n'émergera, car les atomes ne sont pas susceptibles d'être transformés. Et ce que chacun d'eux n'a pas ne se trouvera pas davantage dans le résultat de leur addition : un zéro de chaleur plus un zéro de chaleur ne donnera pas un quelconque degré de chaleur. De même pour la vie. ${ }^{4}$ A fortiori, la connaissance ne saurait être expliquée par des rencontres d'atomes épicuriens :

Quant à la sensation, à l'âme, à l'intellect et à la pensée, [les épicuriens] ne donnent pas même à qui le voudrait une idée de la façon dont ils pourraient

2 «Epicure », rem. F.

3 Pros Coloten, 1111 C-D, p. 214.

4 Ibid., 1111 E, p. 216. 
naître du vide et des atomes, dont la conjonction ne suffit à leur donner ni qualité ni affection ou changement : en effet, leur rencontre ne produit ni fusion ni mélange ni coalescence, car elle ne produit que chocs et rebonds. ${ }^{5}$

C'est très exactement cette critique que Bayle reprend à son compte contre tous les épicuriens, anciens et modernes, et qu'il juge définitive : «Qu'on se tourne de tous les côtez imaginables, comme ont fait Lucrece et Gassendi, pour soudre cette difficulté, on ne pourra même pas l'effleurer [...] ${ }^{6}{ }^{6}$

La seule solution, pour les atomistes, est donc de supposer que leurs atomes possèdent originairement la propriété de connaître. En ce cas, un assemblage de ces corpuscules pourra effectivement être doté lui aussi de connaissance. En d'autres termes, le dilemme est : ou bien pas de pensée, ou bien de la pensée $a b$ initio.

De plus, tous les atomes étant supposés avoir mêmes propriétés et leurs rencontres étant fortuites, on ne peut se contenter de supposer que certains soient pensants et d'autres pas. Il faut donc que tous le soient.

Bayle peut alors conclure que, si un atomiste veut rendre compte de l'existence de la pensée, il est obligé de suivre Démocrite et non pas Épicure, c'est-à-dire de recourir au postulat suivant :

[P 1] Chaque atome est, par nature, doué de pensée.

Cela n'est évidemment pas démontrable. Il ne s'agit que d'une pure assomption, destinée à donner la raison de certains effets observables. Mais comment devons-nous interpréter la démonstration de Bayle ? Cette assomption est si étrange qu'il est difficile de ne pas voir là une tentative de discréditer l'atomisme. D'un autre côté, dans l'article « Leucippe » (texte principal), plus que de les acculer à une hypothèse impossible, Bayle semble sincèrement déplorer que les atomistes n'aient pas eu la bonne idée de supposer les atomes animés. Toutefois, la première impression (celle d'un discrédit) se renforce lorsque le matérialisme en général (non pas seulement atomistique) se voit contraint par Bayle d'adopter un postulat similaire, d'où découlent des conséquences tout aussi étranges.

\section{Dicéarque et le cadavre vivant}

Dicéarque était un disciple d'Aristote, ce qui veut dire selon toute probabilité que la matière était pour lui composée d'éléments qualitativement 
différenciés et non d'atomes. Mais il a nié que l'âme fût quelque chose de distinct du corps. Elle ne serait, d'après lui, selon le passage de Cicéron que cite Bayle, ${ }^{7}$ qu'une « force » (vis) corporelle également distribuée dans tous les corps vivants, humains ou animaux, dont elle est inséparable. Il semble que, toujours selon Dicéarque, cette « force » résulterait de l' « harmonie » des éléments constitutifs du corps. En d'autres termes, il n'existe que des corps, dont certains cependant sont configurés de telle manière qu'ils possèdent puissance de croître et sensation.

Bayle oppose à Dicéarque ce qui est au fond un renforcement et une extension de la conclusion précédente : si l'âme ne fait « qu'un seul et simple être avec les corps qu'on nomme vivans ", dès lors cette force se trouve originairement dans tous les corps, quels qu'ils soient. ${ }^{8}$ Le dilemme devient : ou bien pas de pensée, ou bien de la pensée partout.

La démonstration [argument 2] qu'utilise Bayle pour établir cette alternative repose en premier lieu sur le principe :

[2a] Ce qui n'est point distinct du corps est essentiellement le corps.

Comme Todd Ryan 1'a bien mis en lumière, ${ }^{9}$ Bayle rejette la distinction scolastique entre d'une part accidents proprement dits et d'autre part propres ou accidents inséparables, c'est-à-dire propriétés toujours et nécessairement conjointes à l'essence d'une substance, mais qui ne constituent pas l'essence de cette substance. Pour Bayle, une propriété inséparable fait tout simplement partie de l'essence. Dès lors, ou bien une propriété est un simple accident contingent, séparable et distinct de la chose, ou bien elle est un attribut essentiel.

Or, Dicéarque affirme que l'âme n'est point distincte des corps vivants mais en est une propriété essentielle. Par conséquent, il est obligé d'en dire autant de tous les corps en général car :

[2b] Si une propriété est essentielle, elle appartient aux corps en tant que corps.

Il faut donc ou bien accorder les même facultés à tous les corps et parties de corps, ou bien ne les accorder à aucun. Puisque de fait il y a de la pensée en certains êtres (tels que nous-mêmes, à l'évidence), opter pour la seconde branche de cette alternative conduit à admettre que ces êtres ne sont pas des corps, c'est-à-dire que « la substance qui pense est distincte du corps ». Si l'on choisit la première branche de l'alternative, le fait que la matière soit pensante

7 « Dicéarque », rem. C.

8 Ibid.

9 Pierre Bayle's Cartesian Metaphysics, p. 59-60. 
en certains êtres implique qu'elle doive l'être partout. Le matérialisme ne peut donc être qu'un panpsychisme. ${ }^{10}$

Mais Bayle n'en reste pas là. Il montre que la logique du matérialisme doit conduire à endosser des conséquences si bizarres qu'en comparaison la seconde branche de l'alternative, c'est-à-dire le dualisme corps/esprit, paraît d'une simplicité lumineuse. En effet, Bayle poursuit son raisonnement en introduisant ce principe :

[2c] Une propriété essentielle ne peut être jamais perdue.

Qu'il soit dans un corps animal ou un corps inerte, un corpuscule reste le même corpuscule. Il faudra donc, dit Bayle, admettre que la faculté de connaissance est encore présente dans les cadavres. ${ }^{11}$ Il faudra admettre aussi que les corpuscules libérés par la dissolution du corps emportent chacun avec soi sa vie et son âme, car "si un corps est capable de douleur lors qu'il est placé dans les nerfs, il l'est aussi en quelque endroit qu'il se trouve, ou dans les pierres, ou dans les métaux, ou dans l'air, ou dans la mer $\gg .^{12}$

En bref, qui a pensé pensera. Cela vaut de tous les corps. Et cette propriété leur appartient par nature. Par conséquent, dans un matérialisme cohérent l'attribut pensée doit être universel et indestructible. Au total, Bayle accule donc le matérialiste à endosser le postulat suivant :

[P 2] Tous les corps pensent, et pensent toujours.

\section{La continuité des modes}

Ayant décoché sa flèche, Bayle se rend compte qu'une objection lui sera adressée, qui attaquera la première prémisse de son raisonnement, à savoir que [2a] « ce qui n'est point distinct du corps est essentiellement le corps ». La pensée, dira-t-on, est « une modification du corps ». ${ }^{13}$ Il faut entendre, dans cette objection, " modification » au sens technique, cartésien, de " mode », c'est-à-dire une variation particulière fondée sur une essence commune, comme l'est par exemple la forme d'un objet à l'égard de l'extension. Il est entendu qu'un mode n'est pas un accident, propre ou non, au sens scolastique, c'est-àdire une entité distincte de la substance. Mais il n'est pas non plus purement et simplement identique à l'essence. Sinon, autant dire que tous les corps ont la même forme. Donc, puisqu'un morceau de cire peut être carré puis rond

$10 \mathrm{Cf}$. « Lucrèce », rem. F.

11 « Dicéarque », rem. C. Voir aussi ibid., rem. L.

12 «Dicéarque », rem. C.

$13 \mathrm{lbid}$. 
sans cesser d'être le même morceau de cire, le matérialiste pourra arguer : la matière seule existe, et partout la même, mais le sentiment n'apparaît comme mode que dans des corps qui sont organisés d'une manière bien précise. ${ }^{14}$

Bayle ne se laisse pas démonter par cette objection qui semble de bon sens. Au contraire, il trouve la proposition «absurde». Il lui oppose en effet un troisième argument, fondé sur le principe de l'homogénéité et de la continuité des modes. Il est entendu que le morceau de cire peut être carré puis rond, mais une forme est remplacée par une autre forme, pas par un son, et la cire ne demeure pas sans forme. On peut exprimer la même chose dans les termes de la physique aristotélicienne. Il est bien vrai que le changement va d'un contraire à l'autre, par exemple du froid au chaud, ou du noir au blanc, mais du moins les deux extrêmes appartiennent au même genre des qualités tactiles ou des couleurs. Certes, apparemment certains contraires semblent être tels que l'un est positif et l'autre seulement une «privation » : par exemple mouvement et repos, si le repos n'est que l'absence de mouvement. Mais en fait, chacun de ces deux extrêmes est une modalité de présence locale : le repos est la permanence d'une même présence locale, le mouvement la continuelle acquisition de nouvelles présences locales. Lorsqu'il cesse de se mouvoir, un corps ne perd pas toute présence locale, il ne fait que conserver la même. On voit donc que l'on peut toujours ranger les modes successifs d'une chose sous un même genre.

Tant l'ancienne que la nouvelle philosophie approuvent donc ce double réquisit :

[3a] Il ne peut y avoir de solution de continuité dans la succession des modes (un corps ne peut se trouver sans modalité) ;

[3b] Les modalités successives doivent être de même nature.

Le principe de l'homogénéité et de la continuité des modes peut donc être exprimé ainsi :

[3c] Une modalité ne peut disparaître que si elle est remplacée par une autre modalité de même genre.

Dès lors, Bayle peut porter l'estocade :

[...] il n'y a point de sentiment qui soit chassé de sa substance que par l'introduction de quelque autre sentiment. [...] si les esprits animaux n'ont pas hors des nerfs le sentiment qu'ils y avoient, ils ne l'ont perdu qu'en aquérant une autre sorte de sentiment. 
Certes, il est possible que ce soit une tout autre forme de sentiment, dont nous ne savons rien. Mais, comme le dit Bayle, « rien n'empêche que le sentiment ne soit un genre qui ait au dessous de soi d'autres genres, avant qu'on arrive à ce qu'on appelle species infima». Autrement dit, il se peut que la nouvelle forme de conscience soit opposée à l'ancienne tout autant que le blanc l'est au noir ou le carré au cercle, mais cela sera tout de même une espèce incluse dans un genre plus vaste, que nous pouvons nommer « faculté de sentir » ou bien appeler d'un autre nom, peu importe, et qui impliquera une communauté générique entre les deux modalités.

Bayle peut donc maintenir que le principe [2c] entraîne nécessairement qu' " un corps qui sentiroit une fois, sentiroit toujours ». Même si elle n'est qu'une modalité des substances, et non pas directement leur essence, la pensée est comme la présence locale : elle doit être possédée sous une forme ou sous une autre.

Par conséquent, le fait qu'une partie corporelle soit dissociée d'un ensemble qui pense n'implique pas qu'elle perde la propriété de penser. $\mathrm{Au}$ contraire, elle ne peut pas la perdre, en vertu du principe suivant :

[3d] Le mode est possédé par les parties comme par le tout.

Par exemple, toutes les parties d'un corps en mouvement sont en mouvement. De même, si la partie a pensé avec le tout, prise isolément elle ne peut que conserver cette propriété (éventuellement sous une autre forme).

Bayle peut donc conclure que la version modale du matérialisme est obligée de postuler que :

[P 3] La pensée étant un mode d'un corps, ce corps conservera toujours la pensée sous une forme ou sous une autre.

Si l'on veut éviter cette conséquence onéreuse, il faudra au contraire nier que la matière puisse jamais posséder, ne serait-ce qu'un instant, la faculté de penser.

\section{Toland et les machines pensantes}

Une objection venant de John Toland ${ }^{15}$ a donné un prétexte pour ajouter à l'article «Dicéarque » une remarque supplémentaire (L) et compléter l'argument précédent.

Les principes [2c] et [3c] permettaient de procéder, si je puis dire, de haut en bas : une fois admis qu'un corps possède une propriété, on ne peut plus la 
lui faire perdre ; par conséquent, il est impossible de régresser de la matière pensante à la matière non-pensante.

Maintenant, Bayle va en sens inverse démontrer qu'on ne peut pas passer de la matière non-pensante à la matière pensante. C'est un quatrième argument, dont le principe est identique à l'objection de Plutarque [argument 1] utilisée dans l'article « Epicure », mais qui sera ici exprimé en termes cartésiens.

Toland a proposé une instance visant à montrer que Dicéarque n'est pas obligé de concéder que toute matière est toujours et partout pensante. De même qu'une machine ne fonctionne que si ses pièces sont disposées d'une certaine façon, à l'exclusion des autres, la matière n'est sensible que lorsque ses parties sont arrangées d'une certaine manière. Dans le système de Descartes, rappellet-il, un chien ne diffère pas d'une pierre parce qu'il aurait une âme : «c'est uniquement en ce qu'il est composé de parties tellement rangées qu'elles sont une machine, ce que l'arrangement des corpuscules d'une pierre ne fait pas ». Dicéarque ne dit rien de plus, sinon qu'il fait de l'homme une machine aussi, « d'où il résultera que l'âme humaine n'est point distincte du corps, mais qu'elle est seulement une construction, une disposition machinale de plusieurs parties de matière ». On n'est nullement contraint d'avouer que cette propriété demeure dans un assemblage différent, un cadavre par exemple.

En somme, Toland soutient que certaines propriétés n'appartiennent qu'au tout organisé, non aux parties séparées (contrairement à [3d]) ${ }^{16}$ ou disposées dans un ordre différent. Nous revenons donc à la question de savoir si les pièces de la machine, chacune dépourvue de pensée, peuvent faire un être pensant une fois mis en présence les unes des autres selon un certain ordre. Bayle, pour sa part, a déjà répondu par la négative dans la rem. C, grâce aux arguments [2] et [3]. Mais il propose ici un argument supplémentaire [4], fondé purement et simplement sur le dualisme cartésien de la pensée et de l'étendue:

[4a] Tous les effets de changements dans l'étendue se trouvent dans l'étendue.

[4b] L'on ne trouve dans les effets de changements dans l'étendue aucune des caractéristiques de la pensée, ni dans les effets des changements de la pensée aucune des caractéristiques de l'étendue.

[4c] On ne fera jamais de la pensée avec de l'étendue.

La proposition [4b] repose naturellement sur le fait que les changements dans l'étendue ne consistent que dans du mouvement, dans les divers déplacements et modifications de particules de matière, et que l'on ne constate 
rien là qui évoque les faits de conscience. C'est une thèse cartésienne que Bayle enseignait déjà à Sedan, ${ }^{17}$ et qu'il maintient ici.

Bayle peut alors accepter la comparaison du corps avec une machine qui ne fonctionne que si ses pièces sont convenablement assemblées. Mais il retourne cette analogie à son avantage.

Tout d'abord, « tout ce que peut faire l'arrangement de ces organes se réduit comme dans l'horloge à un mouvement local diversement modifié ». Les rouages ne font que transmettre du mouvement et n'introduisent de variation que du plus au moins, c'est-à-dire des changements non qualitatifs, qui ne donnent pas lieu à émergence de nouvelles propriétés.

Ensuite, avant d'être placé d'une certaine façon, chacun des rouages doit déjà être par lui-même constitué d'une étendue impénétrable. C'est la condition pour que, poussé par une certaine force, il transmette à son tour le mouvement. Ce n'est pas l'arrangement des pièces qui leur confère l'extension. De même, conclut Bayle, « l'arrangement des organes du corps de l'homme ne serviroit de rien pour produire la pensée si chaque organe avant que d'être mis à sa place n'avoit actuellement le don de penser $»$.

Autrement dit, encore une fois ce qui apparaît à l'arrivée doit déjà être donné au départ. Sur un fondement cartésien cette fois, Bayle refuse de nouveau l'émergence de la pensée à partir du non-pensant, de la sensibilité à partir du non-sensible, etc. Bayle peut par conséquent confirmer que Dicéarque aurait dû postuler que la vie, la sensibilité, la pensée sont présentes d'emblée dans la matière. Décidément, le matérialisme, atomiste ou non, ne peut être qu'un panpsychisme.

\section{Leucippe, Locke, et le cartésianisme de Bayle}

De nouveau, on pourrait se demander si cet avis donné aux matérialistes n'est pas un véritable conseil d'ami et si, après tout, ce panpsychisme n'est pas viable aux yeux de Bayle. Mais la fin de la rem. E, dans l'article « Leucippe », permet d'en douter. Après avoir de nouveau recommandé aux atomistes d'adopter l'hypothèse "démocritéenne », Bayle ramène cette hypothèse au même degré de vraisemblance, ou plutôt d'invraisemblance, que celle que les atomistes s'étaient accordé à titre principiel :

ils n'avoient pas moins le droit de supposer des atômes animez que d'en supposer d'incréez et de leur donner la vertu motrice. 
Ils n'en avaient pas moins le droit, mais on peut dire aussi qu'ils n'en avaient pas plus le droit :

Il est aussi malaisé de concevoir cette vertu [motrice] dans un atôme que d'y concevoir le sentiment. L'étendue et la dureté remplissent dans nos idées toute la nature d'un atôme. La force de se mouvoir n'y est pas comprise ; c'est un objet que nos idées trouvent étranger et extrinseque à l'égard du corps et de l'étendue, toute de même que la connoissance.

Bayle se montre ici encore cartésien en ce qu'il ne voit comme propriétés du corps, par idées claires et distinctes, que l'étendue et l'impénétrabilité. Une force motrice inhérente à la matière, tout comme la conscience, serait une virtualité cachée, comparable, et comparée par Bayle, à la vertu motrice attribuée par Aristote à l'âme.

Dans la même ligne cartésienne, après avoir affirmé dans l'article « Dicéarque ", rem. L, que personne «n'a osé dire jusqu'ici qu'il concevoit clairement qu'afin de faire passer une substance de la privation de toute pensée à la pensée actuelle, il suffisoit de la mouvoir », Bayle insiste fortement et ajoute : " quand même quelques uns se vanteroient de concevoir cela clairement, ils ne mériteroient point d'être crus ». Pour faire taire ces « obstinés », il faut leur opposer, dit-il, un certain passage d'Aristote, qui n'est autre que celui où le Stagirite énonce le principe de non-contradiction et dit que la même chose ne peut pas à la fois être et n'être pas. ${ }^{18}$ Cela revient à ajouter un nouvel argument :

[5] Il y a contradiction à faire coexister pensée et matière dans un même sujet.

Par cet argument, Bayle ramène à un pur non-sens la prétention de concevoir clairement comment l'étendue engendrerait de la pensée. Ceux qui ont cette prétention ne savent en réalité pas ce qu'ils disent, puisque cela consiste à affirmer qu'une même chose en même temps possède et ne possède pas un ensemble de propriétés telles que celles qui caractérisent la pensée. ${ }^{19}$ Matière et pensée ont des caractéristiques incompatibles, de sorte qu'elles ne peuvent se trouver que dans des sujets numériquement distincts. Parmi les caractéristiques de la matière sont la divisibilité et le fait d'occuper un espace ; parmi les caractéristiques de la pensée sont l'indivisibilité et le fait de n'occuper aucun espace. Les deux ne peuvent donc être réunis dans une même substance. 
Que la matière pense est donc posé comme contradictoire, c'est-à-dire impossible, ce qui est plus que l'incompréhensibilité actuelle pour notre esprit que Locke admet mais qu'il juge surmontable par un argument ex omnipotentia divina : sans que nous puissions savoir comment, Dieu pourrait adjoindre à de la matière la faculté de penser. ${ }^{20}$ Pour rejeter la possibilité de la matière pensante, plaide Locke, il faudrait démontrer positivement qu'il y a contradiction, car ce qui est incompréhensible (de notre point de vue) n'est pas forcément impossible, alors que ce qui contient une contradiction patente l'est. Mais c'est justement cela qu'affirme Bayle : qu'il y a une contradiction patente, et que la matière ne peut donc en aucun cas penser.

Certes, Locke peut toujours se défendre en disant que pour lui l'extension n'est pas l'essence de la matière, que nous ne savons pas ce qui fait la nature d'une substance, et que c'est pour cela qu'il n'y a pas nécessairement contradiction avec la pensée : ces deux propriétés pourraient coexister dans une substance dont nous ne connaissons pas l'essence. En d'autres termes, une dualité de propriétés qui ne sont pas réductibles l'une à l'autre n'implique pas nécessairement une dualité de substances. Mais ce n'est pas ce que croit Bayle pour sa part, même à une date tardive :

[...] nous savons que les attributs essentiels d'une substance ne diférent point numériquement entre eux, et ainsi nous ne saurions croire qu'il est possible que la matiere s'allie avec l'étenduë par un attribut et avec la pensée par un autre. ${ }^{21}$

Fondamentalement, le différend de Bayle avec Locke sur ce point tient à ce que Bayle est resté inébranlable dans son adhésion à deux thèses cartésiennes :

[a] Il n'y a qu'un attribut principal par substance.

[b] L'étendue est cet attribut pour la matière, c'est-à-dire est l'essence de la matière. ${ }^{22}$

C'est pour cela que Bayle soutient qu'il y aurait contradiction à imaginer une chose qui simultanément posséderait et ne posséderait pas les caractères de l'étendue. La conséquence en est que Dieu même ne pourrait faire qu'une

20 Essay IV, iii, 6, vol. 2, p. 193. Cette hypothèse de Locke et la discussion avec Stillingfleet qui en a résulté sont également expliquées dans « Dicéarque », rem. M. Je tombe d'accord avec T. Ryan (op. cit., p. 35-36), pour dire que, si Bayle a soulevé, par anticipation, l'hypothèse de Locke dans ses objections à Poiret $(O D$ IV, p. 150b-151a), il a cependant changé d'avis par la suite.

$21 R Q P$ III, xv : OD III, p. 942b.

22 Sur l'importance de ces thèses cartésiennes dans la pensée de Bayle, voir T. Ryan, op. cit., p. 11-22. 
matière fût pensante, dans la mesure où Dieu (s'il n'est pas le Dieu cartésien) ne peut réaliser ce qui est contradictoire. ${ }^{23}$

De plus, lorsque Locke dit que nous ne savons pas si la pensée ne pourrait en fait convenir à la matière, il ne s'avance guère sur ce que signifie le verbe "convenir». Mais Bayle est beaucoup plus restrictif. Si une propriété « convient » à un certain sujet, ce n'est pas seulement qu'elle lui est attribuable : « ce qui convient à quelque chose doit être fondé sur son essence et la supposer $»,{ }^{24}$ au lieu d'être arbitrairement juxtaposé à cette essence. Exemple donné par Bayle : les attributs des corps comme être coloré, rond, diaphane, sont « fondez sur l'étenduë et la supposent». Ils ne conviendraient donc pas à des points mathématiques. Autrement dit, il ne suffit pas de lancer une hypothèse gratuite et de répondre : «pourquoi pas ?». Il faut montrer qu'il y a un possibilité réelle de convenance entre la propriété en question et son sujet récepteur. Or, « on conçoit que deux êtres sont d'espèces différentes, quand les attributs essentiels de l'un ne sont pas conçus pouvoir convenir à l'autre $»{ }^{25} \mathrm{Ce}$ qui est le cas pour la pensée et la matière : " l'étenduë, la figure et la divisibilité ne sont fondées sur l'essence de l'âme, ni ne la supposent $»{ }_{,}{ }^{26}$ elles ne peuvent donc convenir à l'âme ; et vice versa. Par conséquent, en aucun cas la pensée ne pourrait être associée à la matière, même surnaturellement.

Bayle fonde donc sa position sur un raisonnement qui est en fait l'inverse de celui qui conduit au panpsychisme. Le matérialiste devrait dire : puisque certains êtres pensent et qu'il sont matériels, toute matière pense. Au contraire, pour Bayle, puisqu'il y a des êtres qui ne pensent pas, « il est certain que la figure et l'étendue» que l'on trouve dans ces êtres «ne supposent point la

23 Cf. la lettre à Shaftesbury du 23 novembre 1699 (OD IV, p. 786). Ce qu'ajoute Bayle dans la rem. C de l'article "Dicéarque ", à la suite du rejet de l'objection modale [arg. 3], me paraît pouvoir contenir également une ligne de défense contre la conception lockéenne de la matière. Le système qu'il réfute, précise Bayle, peut bien prétendre que le mode qu'est le sentiment est fondé « sur quelque attribut de la matière différent des trois dimensions, et inconnu à notre esprit », cela ne change rien à l'affaire : les modifications de cet attribut devront obéir aux même réquisits de continuité et d'homogénéité que par exemple les figures, modes de l'extension, à savoir qu'une modalité doit être chassée par une autre de même nature [3c]. Autrement dit, même si l'essence de la matière nous est inconnue, même si elle recèle des propriétés autres que l'extension, et en particulier la pensée, le tenant de cette hypothèse est acculé à la même conclusion : il doit concéder qu'alors la matière restera toujours pensante, sous une forme ou sous une autre. Or, c'est une conséquence que Locke n'accepterait pas, puisqu'il s'en sert, négativement, dans sa preuve de l'existence nécessaire d'un être immatériel et éternel (Essay, IV, x, 10, vol. 2, p. 314315). Si Bayle veut contrer l'hypothèse de la matière pensante, il me semble donc que sa stratégie n'a pas à reposer exclusivement sur la thèse cartésienne de l'identité entre matière et étendue (et dès lors sur l'« Achille de la psychologie rationnelle "). II pourra montrer que même en admettant que la matière soit autre chose que la simple extension, son adversaire s'engage à des absurdités telles qu'un cadavre pensant.

$24 O D$ IV, p. 455 ; je souligne.

$25 \mathrm{Ibid}$. Je souligne.

$26 \mathrm{Ibid}$. 
faculté de penser »; dès lors, figures et étendue ne « conviennent » jamais à la faculté de penser. Inversement, puisque Dieu connaît, il y a au moins un cas où l'on voit que la faculté de penser ne suppose pas l'étendue, et l'on peut en déduire comme une règle générale qu'elle ne « convient » pas à l'étendue, car si elle lui « convenait » elle la supposerait toujours.

Par conséquent, si les matérialistes peuvent prêter une âme à la matière, c'est uniquement parce que ex falso sequitur quodlibet : à partir du moment où l'on s'accorde l'existence de virtualités cachées, pourquoi ne pas supposer celle du sentiment et de la pensée ? Mais cela n'en fait pas une propriété compréhensible, compatible avec la notion de matière.

Le conseil que donne Bayle aux matérialistes (poser d'emblée la matière comme pensante) semble donc empoisonné. Comme souvent, Bayle se pose en arbitre de la validité des argumentations et de la cohérence interne des positions: si vous voulez être un matérialiste conséquent, voilà ce que vous devriez endosser. Mais cela n'empêche pas que, même ainsi complétées, ces théories restent exposées à des objections principielles et dirimantes telles que l'incompatibilité de la pensée et de l'étendue. Si Bayle pointe ce que tel philosophe ou théologien aurait dû dire, cela ne signifie bien sûr pas qu'il se rallie à leurs thèses.

\section{Références bibliographiques}

LeIBNIz, G. W. Briefwechsel, in Die Philosophischen Schriften, ed. Gerhardt, Bd. III, réimpr., Hildesheim: Olms, 1960.

Locke, J. An Essay Concerning Human Understanding. 2 vols. New York: Dover, 1959.

Plutarque. Pros Coloten, in Moralia, t. XIV, Cambridge (Mass.)-Londres: Harvard University Press, 1967.

Ryan, T. Pierre Bayle's Cartesian Metaphysics. New York-London: Routledge, 2009 . 